\title{
Erratum: Pseudoscalar transition form factors and the hadronic light-by-light contribution to the anomalous magnetic moment of the muon from holographic QCD [Phys. Rev. D 100, 094038 (2019)]
}

\author{
Josef Leutgeb, Jonas Mager, and Anton Rebhan $\odot$ \\ (Q) (Received 27 August 2021; published 24 September 2021)
}

DOI: 10.1103/PhysRevD.104.059903

In Table II the numerical results (in multiples of $10^{-10}$ ) for the HW1 model need to be corrected according to

\begin{tabular}{lcccc}
\hline Model & $a_{\mu}^{\pi^{0}}$ & $a_{\mu}^{\eta}$ & $a_{\mu}^{\eta^{\prime}}$ & Sum \\
\hline HW1 & 6.52 & 1.82 & $1.32 \mid 1.56$ & 9.90 \\
\hline
\end{tabular}

The range of results for $a_{\mu}^{\pi^{0}}$ from the bottom-up holographic models taken together is thus increased from $(5.7 \ldots 6.1) \times$ $10^{-10}=5.9(2) \times 10^{-10}$ to $(5.7 \ldots 6.5) \times 10^{-10}=6.1(4) \times 10^{-10}$. Instead of being at the lower end of the result [1] $\left(a_{\mu}^{\text {dispersive }}=6.3(3) \times 10^{-10}\right)$ obtained in the dispersion theory framework of Ref. [2], this is now only slightly smaller and fully consistent.

\section{ACKNOWLEDGMENTS}

J. L. was supported by the FWF doctoral program Particles \& Interactions, Project No. W1252-N27; J. M. was supported by the FWF Project No. P 33655.

[1] M. Hoferichter, B.-L. Hoid, B. Kubis, S. Leupold, and S. P. Schneider, Dispersion relation for hadronic light-by-light scattering: Pion pole, J. High Energy Phys. 10 (2018) 141.

[2] G. Colangelo, M. Hoferichter, M. Procura, and P. Stoffer, Dispersion relation for hadronic light-by-light scattering: Theoretical foundations, J. High Energy Phys. 09 (2015) 074.

Published by the American Physical Society under the terms of the Creative Commons Attribution 4.0 International license. Further distribution of this work must maintain attribution to the author(s) and the published articles title, journal citation, and DOI. 